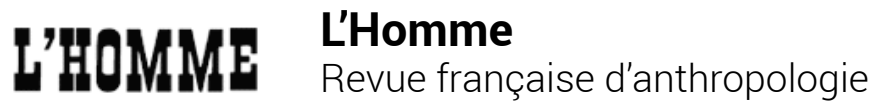

$211 \mid 2014$

Varia

\section{La machine aksak et les fascinantes formules asymétriques du petit luth de Turquie}

\author{
Marc Chemillier
}

\section{(2) OpenEdition}

Journals

\section{Édition électronique}

URL : http://journals.openedition.org/lhomme/23634

DOl : 10.4000//homme.23634

ISSN : 1953-8103

Éditeur

Éditions de l'EHESS

\section{Édition imprimée}

Date de publication : 7 juillet 2014

Pagination : 129-140

ISSN : 0439-4216

\section{Référence électronique}

Marc Chemillier, «La machine aksak et les fascinantes formules asymétriques du petit luth de

Turquie », L'Homme [En ligne], 211 | 2014, mis en ligne le 07 juillet 2016, consulté le 23 avril 2019. URL : http://journals.openedition.org//homme/23634 ; DOI : 10.4000//homme.23634 


\section{La machine aksak et les fascinantes formules asymétriques du petit luth de Turquie}

Marc Chemillier

$\mathrm{L}$

LIVRE de Jérôme Cler sur la musique du petit luth de Turquie témoigne de la grande intimité dans laquelle il se trouve aujourd'hui avec cette petite société située dans les montagnes de Turquie, sur un territoire de quelques kilomètres carrés formé de pâturages (appelés yayla) où il s'est rendu régulièrement pendant plus de vingt ans. La densité de ce terrain est rapportée avec beaucoup de vie par la force de son style et par ses qualités littéraires. La richesse des références que l'auteur mobilise et le cadre conceptuel qu'il développe apportent de surcroît à cet objet la profondeur d'une pensée nouvelle sur le plan théorique. Les concepts de territoire, de rythme et d'espace jouent un rôle central dans son approche et en appellent d'autres, introduits au fil du texte, comme ceux de géo-musicologie (p. 47) ou de devenir-mineur (p. 325). Dans ce contexte, il était naturel que Gilles Deleuze et Félix Guattari soient invoqués (1980, cités p. 157) pour les pages célèbres qu'ils ont écrites sur la déterritorialisation dans leur ouvrage maintes fois cité, Mille Plateaux. Mais Jérôme Cler ne s'arrête pas là et il mentionne, parmi les nombreuses références sollicitées dans son livre, d'autres pages du philosophe Gilles Deleuze beaucoup moins connues, comme celles qui traitent du concept de "galop» (p. 212). Deleuze définit le galop comme la "précipitation des présents qui passent "

À propos de Jérôme Cler, Yayla, musique et musiciens de villages en Turquie méridionale, Paris, Geuthner, 2011 [avec CD encarté]. Cet "À propos" est la concrétisation d'échanges entre Jérôme Cler et moi qui remontent à une vingtaine d'années, et qui se sont traduits plus récemment par sa participation à deux séances du séminaire de l'EHESS «Modélisation des savoirs musicaux relevant de l'oralité" (10 mars 2010 et 6 février 2013). Les vidéos sont accessibles sur la page du séminaire : http://ehess.modelisationsavoirs.fr/seminaire. Les expériences de modélisation avec ordinateur relatées dans cet article ont été réalisées dans le cadre du projet IMPROTECH ( "Technologies et musiques improvisées », ANR-09-SsOC-068). 
(1985 : 123). Il n'est pas de notre ressort ici d'embrasser la conception subtile du temps que Deleuze élabore dans cet ouvrage consacré principalement au cinéma. Mais il faut néanmoins souligner que la "précipitation des présents qui passent " se rapporte, sur le plan musical, et plus particulièrement sur celui du rythme, à un phénomène essentiel que l'on appelle la pulsation. Celle-ci se manifeste quand des événements perçus avec une certaine régularité et à une certaine échelle de temps (quelques secondes) se cristallisent (ou "précipitent " pourrait-on dire au sens de la chimie) pour donner naissance à un calibrage de la musique qui change complètement la perception que l'on en a. Une musique pulsée n'est pas du tout la même chose qu'une musique s'écoulant librement, et cela se traduit de diverses manières dont la plus évidente est la mise en mouvement du corps que la pulsation induit à travers la danse. Or précisément, les musiques qu'étudie Jérôme Cler recèlent, sur le plan du rythme, de la pulsation et de la danse, des propriétés étonnantes liées à leur asymétrie. On pourrait dire que le galop deleuzien y est confronté au boitement. C'est sur ce point que portera notre "À propos ", et il est évident que, ce faisant, il ne rendra pas justice à la variété des approches ethnographique, sociologique, philosophique et littéraire qui s'entrecoupent dans cet ouvrage. Mais, dans la mesure où celui-ci comporte une partie musicologique fort développée, qui soulève des problèmes essentiels de modélisation, nous avons choisi de privilégier cette piste de réflexion.

Du point de vue musical, le petit luth joue de courtes formules instrumentales qui sont répétées en boucle avec quelques variations. Associé au chant ou à un instrument mélodique comme le violon ou le hautbois, il peut accompagner la danse, mais il peut aussi être joué en solo en enchaînant les formules les unes après les autres pour constituer une sorte de suites instrumentales exécutée dans un espace intime, ce que Jérôme Cler appelle le "jeu méditatif» (p. 200 et p. 239 pour le jeu méditatif de Hayri Dev). L'une des caractéristiques les plus frappantes de ces airs est qu'ils sont tous construits sur un rythme appelé aksak (ce qui signifie "boiteux », p. 181) comportant neuf unités groupées dans l'ordre $2+2+2+3$, ou dans l'une de ses permutations circulaires, et qui est répété indéfiniment tout au long de la pièce. Le caractère boiteux du rythme vient du groupe de 3 unités qui rompt en quelque sorte la régularité de la série de 2 unités. Bien que les formules de luth soient d'apparence très simples et reposent sur une combinatoire réduite à peu d'éléments, il est très difficile pour un auditeur non acculturé de battre ce rythme en entendant la partie instrumentale, et de placer correctement le groupe de 3 unités. Cette capacité nécessite un habitus au sens de Bourdieu, c'est-àdire une disposition particulière qui s'obtient par un «travail d'inculcation 
et d'appropriation" (cité p. 282). Jérôme Cler met en relation cette capacité avec la notion d'habitus introduite par Bourdieu. Rappelons que le sociologue définit ainsi une disposition particulière de l'esprit obtenue par un «travail d'inculcation et d'appropriation» (Bourdieu 1972: 282). La nécessité d'un habitus pour reproduire certains rythmes asymétriques s'applique à la plupart des rythmes des Balkans, notamment les rythmes bulgares étudiés par le compositeur Béla Bartok à propos desquels Jérôme Cler souligne en effet "l'impossibilité, pour les musiciens rompus au solfege occidental, d'acquérir de nouveaux habitus pour jouer ses rythmes bulgares" (p. 196).

Le caractère insaisissable de ces rythmes associé à la sonorité métallique

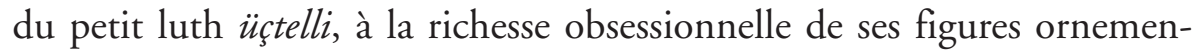
tales et à l'harmonie abrupte de ses séries de quintes ${ }^{1}$ successives, expliquent le pouvoir de fascination que cet instrument est capable d'exercer sur l'auditeur.

Au fil des pages, principalement dans la deuxième partie de l'ouvrage, Jérôme Cler dévoile avec beaucoup de clarté l'économie de ce répertoire musical, en abordant de façon méthodique ses différentes composantes musicologiques que nous allons brièvement rappeler ici. Il précise tout d'abord, dans le chapitre intitulé "Rythme», le sens donné à certains termes descriptifs. Ce chapitre n'est pas seulement illustratif des particularités rythmiques du petit luth de Turquie, c'est aussi une contribution théorique au concept même de rythme. Contrairement à la tradition africaniste établie depuis les travaux de Simha Arom (1985), dans laquelle la "pulsation " et le "temps ${ }^{2}$ désignent une même chose, le rythme aksak étudié par Jérôme Cler conduit à distinguer la pulsation qui correspond aux neuf unités régulièrement espacées sur lesquelles sont fondés ces rythmes (notées en croches dans les transcriptions ci-après), et le temps qui correspond à leurs groupements en $2+2+2+3$. Les temps sont donc irréguliers selon qu'ils correspondent à 2 ou 3 unités, c'est-à-dire à deux durées distinctes (transcrites en noires et noires pointées). Notons qu'audessous du niveau de la pulsation, il existe parfois un niveau supplémentaire de subdivision. Les temps imposent certaines contraintes d'ordre gestuel dans le jeu du luth. En effet, parmi les mouvements de la main

1. La quinte est l'intervalle séparant cinq notes consécutives de la gamme, par exemple do-sol (la gamme étant do-ré-mi-fa-sol-si). Quand les deux extrémités d'une quinte sont jouées simultanément, la sonorité obtenue est réputée avoir une certaine "dureté ». Le petit luth de Turquie joue souvent des séries de quintes successives comme on le verra plus loin.

2. Le mot «temps " est entendu ici au sens d'une unité de durée, comme quand on dit "mesure à quatre temps». 
droite qui pincent les cordes alternativement vers le bas (B) ou vers le haut $(\mathrm{H})$, les temps correspondent toujours à un mouvement vers le bas. Par exemple, une suite continue de pulsations sera réalisée en alternant $(\mathrm{B}+\mathrm{H})+(\mathrm{B}+\mathrm{H})+(\mathrm{B}+\mathrm{H})+(\mathrm{B}+\mathrm{H}+\mathrm{H})$. Mais bien entendu, cette alternance sera modifiée en fonction des durées utilisées comme on peut le voir dans le tableau synthétique présenté plus loin dans le livre (p. 234), qui regroupe les principales séquences de durées que l’on trouve dans la partie du luth.

Le rythme est lié à la danse et celle-ci renforce l'une de ses caractéristiques fondamentales, sur laquelle on reviendra dans la suite de cet article. Il apparaît en effet que deux positions du cycle à neuf pulsations sont affectées d'un poids particulier, la première et la cinquième pulsations. Cela se traduit, dans le mouvement des danseurs, par un processus en deux phases de tension et de détente autour d'un climax situé sur la cinquième pulsation. D'abord les bras sont levés jusqu'à ce point, puis ils se baissent jusqu'au groupe de trois finales (p. 187). On le voit très bien sur la vidéo donnée en référence pour illustrer cette idée (vidéo $\mathrm{n}^{\circ} 10 \mathrm{du}$ DVD), qui montre le spectacle exaltant d'un groupe d'hommes assis en plein air qui se lèvent spontanément au son de la musique et se mettent à danser en cercle dans un champ. On entend le claquement de leurs doigts sur les trois premiers temps (donc jusqu'à la cinquième pulsation), puis ils baissent les bras et s'accroupissent pendant les trois pulsations finales. À 1'08, le groupe se resserre et au moment précis de la cinquième pulsation, les danseurs se dispersent brusquement dans un mouvement qui évoque la figure d'une gerbe. Signalons enfin sur le plan du tempo que ces danses sont plutôt modérées. Il existe trois types de tempo dans le répertoire de cette région qui répartissent les pièces en trois classes distinctes (p. 195) : les pièces lentes appelées zeybek (entre 35 et 150 pulsations par minute), les pièces de tempo moyen appelées sipsi, du nom de la clarinette avec laquelle on les joue (entre 200 et 250 pulsations par minute), et les pièces très rapides teke (entre 350 et 450 pulsations par minute). Le fait remarquable est qu'elles sont toutes fondées sur le même groupement $2+2+2+3$, mais joué à des vitesses différentes, ce qui a pour effet de changer radicalement la perception qu'on en a. C'est principalement aux sipsi que nous nous intéresserons ici.

Après le rythme, Jérôme Cler aborde le système des hauteurs et s'intéresse à la manière dont celui-ci est réalisé sur le luth. Cet aspect fait l'objet du chapitre intitulé "Petit traité du baglama ", bağlama étant le terme qui désigne le luth, üçtelli signifiant «trois cordes». Celles-ci sont accordées sur les hauteurs ré-do-sol (p. 232) et définissent le mode ré-mifa-sol-la-si-do-ré (appelé «mode de ré»). On notera que les cordes ne sont 
pas placées dans l'ordre des hauteurs croissantes puisque la hauteur de celle du haut (ré) est intermédiaire entre les hauteurs des deux du bas do et sol, de sorte que c'est la corde la plus grave do qui se trouve au milieu. Jérôme Cler définit ensuite, de manière synthétique, les trois règles qui déterminent le jeu du bağlama (p. 233). Suivant la première règle, les cordes de do et de sol, qui sont très rapprochées sur le manche, sont souvent jouées ensemble par la main gauche produisant une série de quintes successives, et lorsque ce n'est pas le cas, le mouvement mélodique est réalisé uniquement sur la corde de sol, pendant que celle de do résonne comme un bourdon (en revanche la corde de do n'est pratiquement jamais jouée seule). La deuxième règle est que les principales positions utilisées sur le manche sont les quatre premières donnant les quintes do-sol, ré-la, mi-si, $f a$-do (positions notées $0,1,2,3)$. Enfin, la troisième règle concerne la corde du haut (corde de ré) qui est pressée avec le pouce de la main gauche quand celle-ci est en position 3 (quinte $f a-d o$ ) pour jouer la note fa. Corollaire de la règle des quintes (règle 1 ), la succession peut s'entendre mélodiquement de plusieurs manières différentes, selon que l'on privilégie dans chaque quinte la note du haut ou celle du bas (ainsi le mouvement de la quinte $f a$-do à la quinte do-sol peut s'entendre mélodiquement comme fa allant à do ou fa allant à sol).

Ce chapitre reprend ensuite la question du geste de la main droite et de ses contraintes rythmiques, en donnant un tableau synthétique (p. 234) des principales durées utilisées selon les positions dans le cycle (pulsations de 1 à 9). On constate que les deux seules pulsations sur lesquelles on peut trouver une durée longue (transcrite en noire) sont la première et la cinquième, ce qui corrobore l'observation faite plus haut concernant le mouvement des danseurs qui accentue la cinquième pulsation. Le «Petit traité du bağlama" se poursuit avec la mise en relation de la partie de luth et de la mélodie correspondante jouée sur le violon ou le hautbois. Dans la série de quintes successives, la mélodie prélève librement les notes supérieures ou inférieures pour créer un mouvement "oblique " (transcrit en utilisant des têtes de note plus épaisses pour souligner la note prélevée dans chaque quinte, p. 238). Ce phénomène est sans doute assez courant dans les musiques traditionnelles à travers le monde, et il est attesté en Afrique centrale dans le répertoire des harpistes nzakara sur lequel j'ai travaillé (voir Chemillier 2007 : 154). Il implique que plusieurs mélodies peuvent s'adapter à une même partie instrumentale, selon la manière dont on réalise le mouvement oblique, et la partie instrumentale apparaît donc comme un réservoir de mélodies potentielles, une sorte de classe d'équivalence qui coagule leur diversité mélodique. La fin du chapitre est consacrée à l'ornementation et aux figures stylistiques que Jérôme Cler 
appelle des tropes. Ce sont des motifs directement reliés à la structure de l'instrument et à certains gestes comme celui qui consiste à faire entendre un contour mélodique sans l'aide de la main droite, uniquement en appuyant certains doigts de la main gauche sur le manche que le musicien confirmé apprend à maîtriser notamment dans le " jeu méditatif ", et "qui permettent de sortir du cadre restreint des quintes parallèles» (p. 245).

Le chapitre suivant aborde la question épistémologique essentielle de l'existence d'un "modèle réduit " de ces airs, sur laquelle on reviendra plus loin. Le répertoire du üçtelli est un foisonnement d'airs à la fois tous différents, mais aussi très semblables du fait de l'espace combinatoire restreint dans lequel ils évoluent, défini par les principes exposés ci-dessus (cadre rythmique limité à neuf pulsations, cadre mélodico-harmonique réduit à quatre positions sur le manche). Jérôme Cler utilise à plusieurs reprises la "métaphore du "troupeau" d'airs éparpillés dans la montagne " (p. 283), métaphore qui provient de ses informateurs eux-mêmes (p. 180). Pour tenter de dégager une logique dans cet éparpillement que l'on pourrait qualifier de "pastoral ", il commence par étudier la catégorie des airs les plus simples qui sont dits "monopériodiques", c'est-à-dire qui se répètent toutes les neuf pulsations. Puis il se tourne vers ceux dont la périodicité est double (répétition toutes les dix-huit pulsations), ce qui fait apparaître un principe de dédoublement selon lequel la plupart de ces airs comportent une symétrie interne constituée d'un antécédent se terminant sur do, puis repris sous forme de conséquent conduisant à la finale ré (p. 253). Pour aller plus loin, Jérôme Cler s'efforce de dégager un modèle des airs qui permettrait de les relier les uns aux autres et de dévoiler les processus d'engendrement de nouveaux airs à partir d'airs connus selon une logique d'association d'idées (pp. 256, 262). Ce modèle est obtenu par réduction de la ligne mélodique, en ne retenant que les notes qui paraissent essentielles. Les musiciens peuvent transmettre ces modèles eux-mêmes de diverses manières. Tout d'abord, il leur arrive de jouer la mélodie de façon simplifiée, comme l'illustre cette anecdote que m'a rappelée Jérôme Cler à propos d'un stage de musique traditionnelle au cours duquel Hayri Dev était intervenu en février 1995 à Bondy en région parisienne (et auquel j'avais participé). Celui-ci avait été amené à jouer une mélodie sur une flûte à coulisse qui se trouvait dans cette école de musique, et comme l'instrument ne lui permettait pas de faire tous les subtils ornements qu'il pratique habituellement sur la clarinette en roseau sipsi, il s'était trouvé contraint de jouer une version dépouillée de la mélodie et donc d'en donner un modèle. L'autre voie pour accéder aux modèles s'appuie sur le jeu du luth. Il existe, en effet, une technique simple de jeu que l'on appelle « jeu droit » qui consiste à procéder strictement en quintes 
consécutives (p. 235). C'est la manière de jouer des débutants et elle permet d'obtenir des modèles avec le minimum de notes et d'ornements.

Voici deux exemples de modèles en notation musicale indiquant la mélodie, complétés par des chiffres sous la portée qui correspondent aux positions des quintes jouées sur le luth comme une sorte de tablature [Fig. I]. On voit clairement la structure rythmique $2+2+2+3$ représentée au début par des croches groupées par deux et par une noire $(2+2+2)$ suivies d'un groupe de trois croches (3). On observe dans ces deux modèles un allongement de la durée (transcrit en noire) qui coïncide précisément avec la cinquième pulsation.

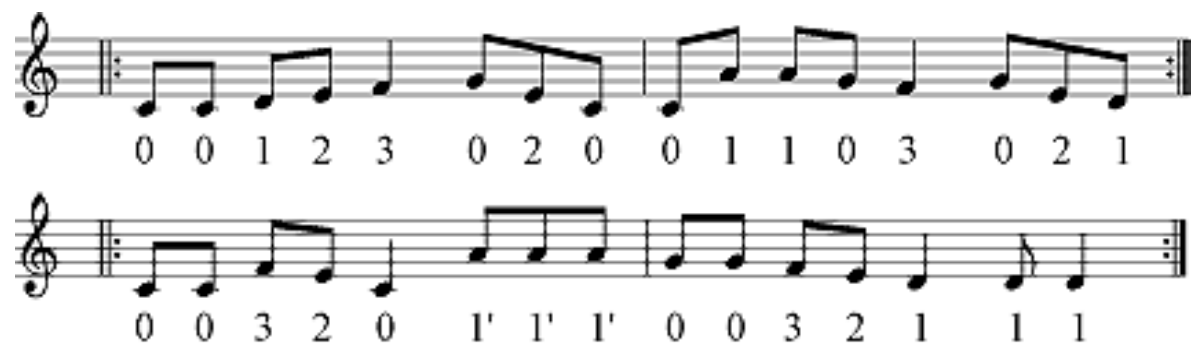

Figure I. Deux formules de luth "modélisées" (c'est-à-dire simplifiées).

Les chiffres indiquent les positions 0, I, 2, 3 sur le manche,

la position I' étant jouée sur la corde du bas seule.

Chaque modèle est en deux parties correspondant à deux énoncés du cycle $2+2+2+3$. Le second modèle a une symétrie créée par la succession de positions 0032 commune aux deux parties. Les deux modèles se terminent sur la finale ré, et le premier a un balancement à mi-parcours sur la note do (position 0) alors que le second termine chacune des deux parties sur la position 1 (le balancement semble reporté en amont sur la cinquième pulsation puisqu'on a 0 la première fois, et 1 la deuxième). Notons que le symbole 1' est utilisé pour distinguer la position 1 jouée en quinte ré-la de la même position jouée uniquement sur la corde du bas avec la note la (la corde du milieu fait alors sonner do à vide en bourdon comme on l'a vu plus haut, "première règle").

Sur le plan anthropologique des savoirs musicaux mis en jeu dans la reconnaissance des rythmes aksak, une question essentielle est posée par le processus de modélisation. Le modèle " réduit " garde-t-il toujours une trace de la structure rythmique asymétrique qui fonde le répertoire, et si oui, comment se manifeste-t-elle? En d'autres termes, peut-on battre la formule aksak $2+2+2+3$ à la seule audition du modèle? Jérôme Cler s'attaque à ces questions avec beaucoup de discernement, en soulevant le problème du caractère "arbitraire " ou non de la battue : 
«Est-elle un opérateur indépendant de la structure mélodique, venant l'informer de diverses manières? Dans ce cas, le cadrage de cette battue ne dépend que d'un consensus culturel, et non d'une nécessité formelle musicale. Ou bien le lien entre mélodie et battue est nécessaire: cela supposerait que certains degrés de l'échelle seraient toujours accentués, ou que le profil mélodique tout entier imposerait ses accentuations» (p. 266).

Il développe sa réflexion en attirant l'attention du lecteur sur certains cas troublants. Le premier concerne deux airs qui sont pratiquement identiques à un décalage près d'une pulsation (p. 268), d'où il résulte que leur profil mélodique est ambigu et peut être interprété rythmiquement de deux manières différentes. Le second cas surprenant est lié à l'existence de pièces dans le répertoire dont le rythme est inversé $(3+2+2+2$ au lieu de $2+2+2+3$ ) et dont le profil mélodico-harmonique est différent de celui des autres pièces. Or, il se trouve que certaines pièces existent sous les deux formes rythmiques (on en voit deux exemples, p. 265 et p. 269). Ces cas extrêmes montrent que la relation entre la partie de luth et le rythme $a k s a k$ est complexe, et que si l'on admet l'existence d'une grammaire régissant ces relations, celle-ci n'est pas exempte d'ambiguïté. Après tout, c'est aussi le cas de la grammaire d'une langue comme le français. On sait bien que des phrases comme "Pierre dit à Paul qu'il boit trop " sont correctes grammaticalement, mais intrinsèquement ambiguës dans la mesure où l'on ne peut déterminer si c'est Pierre ou Paul qui abuse de la boisson. Mais, de la même façon que la grammaire d'une langue joue un rôle essentiel sur le plan cognitif pour permettre à l'esprit humain de comprendre les phrases qu'il entend, il est probable qu'il existe des règles syntaxiques qui facilitent la détection du rythme $2+2+2+3$ dans les formules de $\ddot{u}$ çtelli et qui expliquent ainsi pourquoi tous les membres d'une communauté le battent de la même manière.

Cette discussion sur la possibilité d'exprimer des règles explicites régissant l'organisation rythmique des formules de üçtelli revient à imaginer une sorte de «machine $a k s a k$ ». L'expression est empruntée à Jérôme Cler (p. 309) qui l'emploie à l'occasion d'une réflexion pénétrante développée dans son livre sur les relations entre la mécanique rythmique de l'aksak, avec sa répétition immuable qui laisse parfois s'échapper certaines configurations atypiques comme on l'a vu plus haut, et le concept de rire tel qu'il est étudié par Bergson. Celui-ci écrivait en effet: "Est comique tout arrangement d'actes et d'événements qui nous donne, insérés l'un dans l'autre, l'illusion de la vie et la sensation nette d'un agencement mécanique ", et Bergson illustrait son propos en donnant l'exemple du diable à ressort qui jaillit de sa boîte (1997 [1899] : 53). L'équivalent musical serait le "surgissement de la variante minimale, qui prête à rire ", 
selon l'expression utilisée par Jérôme Cler : "Un musicien comme Hayri Dev sait quelle variante il faut introduire dans la répétition pour que l'assistance puisse sourire, ou rire légèrement» (p. 309).

Pour prolonger cette discussion, nous proposons de réaliser effectivement une telle machine $a k s a k$. On peut s'y prendre de plusieurs manières, la première consistant à utiliser une méthode de type statistique. C'est la voie suivie par Andre Holzapfel et Baris Bozkurt dans leur étude des schémas rythmiques de la théorie musicale turque qu'on appelle usul (Holzapfel \& Bozkurt 2012). Il s'agit de séquences verbales qui définissent des successions de notes accentuées ou non. L'un des usul de la théorie turque est précisément l'aksak. Sa séquence verbale est "düm te ke düm tek tek» dont les syllabes se placent sur les positions 1, 3, 4, 5, 7, $9 \mathrm{du}$ cycle à neuf pulsations. La syllabe la plus accentuée est "düm » qui tombe sur les positions 1 et 5 ce qui corrobore l'observation faite plus haut selon laquelle, dans l'aksak, ces deux positions sont affectées d'un poids particulier. Holzapfel et Bozkurt se sont intéressés à des compositions musicales construites sur ces schémas rythmiques en faisant l'inventaire, pour chaque position définie par l'usul, des notes de la composition qui tombent effectivement sur cette position. Les résultats montrent qu'il y a globalement concordance: les positions où apparaissent le plus de notes sont celles qui sont accentuées dans l'usul correspondant. Dans l'histogramme obtenu pour l'aksak, la position ayant le plus de notes est 5 , puis viennent ensuite 1 et 7 , puis en ordre décroissant du nombre de notes on trouve 4, 3 et 9. On voit que cela coïncide avec les positions accentuées de l'usul. Cette méthode peut être utilisée en sens inverse pour des pièces dont on ne connaît pas le schéma rythmique. On trace l'histogramme du nombre de notes tombant sur les différentes positions, puis on cherche à le faire coïncider avec l'histogramme théorique défini par l'usul. Le problème des "modèles réduits" du petit luth üçtelli est qu'ils contiennent peu d'informations, et de fait, se prêtent difficilement à un traitement statistique. Par exemple, dans la première formule modélisée ci-dessus, toutes les positions coïncident avec une note sauf la 6 (à cause du prolongement de la note en position 5). En revanche, l'usul comporte plusieurs positions non accentuées $(2,6,8)$ de sorte qu'on ne peut déterminer laquelle correspond à la position vide de la formule d'üçtelli.

Une autre manière d'aborder le problème consiste à procéder de proche en proche, par essais successifs. On peut le faire avec un logiciel capable de produire de la musique selon des règles fixées à l'avance et c'est ce que permet précisément de faire le logiciel d'improvisation ImproteK sur lequel j'ai travaillé dans le cadre d'un projet soutenu par l'Agence 
nationale de la recherche (Nika \& Chemillier 2012). Ce logiciel capte le jeu d'un musicien et se sert des phrases enregistrées pour en improviser de nouvelles dans le même style. Son principe est de recombiner des fragments prélevés dans le corpus mémorisé, de sorte que si l'on donne au logiciel un certain nombre de formules de luth modélisées, il sera capable d'en produire de nouvelles. On peut ensuite les faire entendre à des experts de la culture concernée, et leur demander de les valider en les rejouant sur le luth, ou en les accompagnant avec le rythme aksak frappé à l'aide d'un instrument à percussion. Une première classe de modèles artificiels pourrait être fabriquée, en prélevant certains fragments dans des modèles connus, mais en préservant leur position à l'intérieur du cycle rythmique $2+2+2+3$ (c'est-à-dire qu'un élément en position 1 sera prélevé sur un modèle connu dans la même position 1, et ainsi de suite pour les positions de 1 à 9). Puis on pourrait explorer d'autres classes obtenues en autorisant certaines permutations de positions dans le cycle, puis en poussant le processus à l'extrême, on pourrait autoriser toutes les permutations en considérant les neuf pulsations comme interchangeables. La génération de ces faux modèles délimiterait ainsi différentes classes dans lesquelles on effectuerait en quelque sorte des "coups de sonde ", en tirant au hasard certains éléments pour évaluer leur degré de grammaticalité. On pourrait ainsi, de proche en proche, affiner les hypothèses sur les aspects du profil des séquences qui sont essentiels dans la perception du rythme, et sur ceux qui ne le sont pas et qui peuvent être modifiés sans conséquence.

Une telle expérience a été réalisée lors du séminaire de l'École des hautes études en sciences sociales «Modélisations des savoirs musicaux relevant de l'oralité ", le 6 février 2013. Le logiciel a calculé, puis fait entendre plusieurs modèles artificiels qui ont été évalués par l'expert participant à l'expérience. C'est Jérôme Cler lui-même qui jouait le rôle d'expert car il ne se contente pas d'étudier ce répertoire mais il le pratique également en tant que musicien en l'interprétant sur l'instrument. Très vite l'expérience a révélé certaines contraintes qui pèsent implicitement sur l'organisation des formules. La symétrie entre l'antécédent et le conséquent, c'est-à-dire le fait que les deux parties commencent de la même manière, apparaît comme un élément essentiel pour rendre les modèles acceptables et pour que l'on puisse les accompagner rythmiquement. Curieusement, cet aspect est moins important quand les modèles sont calculés en respectant l'ordre des pulsations (de 1 à 9) que quand on mélange librement les pulsations, car dans ce dernier cas, le critère de symétrie se manifeste comme une sorte de moyen auxiliaire nécessaire pour se repérer à l'intérieur des modèles. D'autres difficultés ont surgi au cours de l'expérience. Par exemple, les modèles commençant par mi-si paraissent bizarres (position 
2). Ou encore, la position 3 ( $f a-d o)$ comme fin de l'antécédent répondant au conséquent n'est pas une terminaison correcte. Voici le modèle artificiel qui a conduit Jérôme Cler à formuler cette dernière remarque :

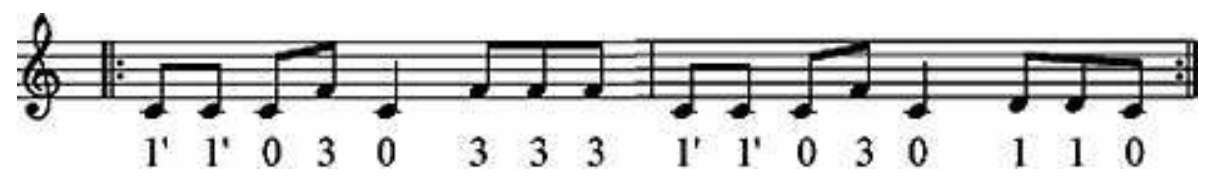

Figure 2. Un modèle artificiel engendré par le logiciel ImproteK.

On voit que le modèle comporte une symétrie (1'1'030 répété deux fois), et qu'il possède les allongements attendus de la durée sur la cinquième pulsation (noires dans la transcription). De plus, bien que la terminaison ne soit pas strictement sur la finale ré (puisque la dernière note est $d o$ ), le ré est malgré tout suffisamment présent dans le groupe de trois pulsations finales (110). En revanche, ce qui gêne dans ce modèle, c'est la présence de $f a-f a-f a$ à la fin de l'antécédent (333), qui n'apparaît pas comme une solution satisfaisante pour le repos qui se trouve généralement à cet endroit.

À la fin de son livre, Jérôme Cler s’interroge sur le devenir de ces airs dans le contexte de la globalisation. Le dernier chapitre intitulé « Le village et la société globale " revient, non sans une certaine émotion qui affleure derrière les mots, sur le parcours de deux musiciens avec lesquels il a travaillé, Ramazan Güngör et Hayri Dev. Le premier s'est exilé en ville et a eu une certaine influence sur plusieurs musiciens à l'échelle macrorégionale et nationale, qui lui ont rendu hommage lors d'une fête organisée pour ses soixante-dix ans. Mais cet hommage était teinté d'une certaine tristesse, Ramazan était vieux, ne jouait plus qu'avec peine, et ses disciples étaient devenus beaucoup plus connus que lui. L'autre musicien était resté au village et ne s'était guère soucié de transmettre quoi que ce soit de son art. Mais l'ironie de l'histoire a voulu que ce soit lui qui reçoive, il y a quelques années, une consécration internationale en étant désigné "trésor humain vivant " par l'Unesco. Le travail de l'ethnomusicologue qui l'a côtoyé pendant plus de vingt ans n'y est pas étranger. Les réactions d'Hayri Dev à cet événement, dans lesquelles c'est l'humour qui prévaut, donnent à Jérôme Cler l'occasion de reprendre le fil de sa réflexion sur le rire évoquée plus haut et sur le concept de devenir-mineur qu'il développe dans ces dernières pages. Hayri Dev est « tout entier dans l'acte, jouant, dansant, affirmant son propre devenir-mineur par la fine 
pointe de l'humour» (p. 328). Il n'y a pas de revendication identitaire dans l'attitude de Hayri Dev, "jamais un "être", jamais une ontologie, mais toujours un devenir dans lequel l'ethnomusicologue et son terrain étaient joyeusement emportés »(p. 326). Face à ce que Jérôme Cler appelle les "pratiques du monde majeur» (p. 329), le devenir-mineur se caractérise, en fin de compte, par une "aimable irrévérence ".

École des hautes études en sciences sociales Centre d'analyse et de mathématique sociales, Paris chemilli@ehess.fr

MOTS CLÉS/KEYWORDS : ethnomusicologie/ethnomusicology - petit luth de Turquie/small lute of Turkey - aksak - musique traditonnelle/traditional music.

\section{RÉFÉRENCES CITÉES}

Arom, Simha

1985 Polyphonies et polyrythmies instrumentales d'Afrique centrale.

Structure et méthodologie. Paris, SELAF.

Bergson, Henri

1997 [1899] Le Rire.

Paris, Presses universitaires de France.

Bourdieu, Pierre

1972 Esquisse d'une théorie de la pratique, précédée de Trois études d'ethnologie kabyle. Genève, Droz.

Chemillier, Marc

2007 Les Mathématiques naturelles. Paris, Odile Jacob.

Deleuze, Gilles

1985 Cinéma, 2. L'image-temps. Paris, Minuit.
Deleuze, Gilles \& Félix Guattari

1980 Capitalisme et schizophrénie, 2. Mille plateaux. Paris, Minuit.

\section{Holzapfel, Andre \& Baris Bozkurt}

2012 "Metrical Strength and Contradiction in Turkish Makam Music", Proceedings of the 2ND CompMusic Workshop, Istanbul, Turkey, July 12-13, 2012 : 79-84 [http://repositori.upf.edu/handle/10230/20 426].

Nika, Jérôme \& Marc Chemillier 2012 «ImproteK : intégrer des contrôles harmoniques pour l'improvisation musicale dans la filiation d'OMax ", Actes des journées d'informatique musicale (JIM 2012), Mons, Belgique, 9-11 mai 2012: 147-155. [http://articles.ircam.fr/textes/Nika12b/ index.pdf]. 